\title{
e-Migrinter
}

$18 \mid 2019$

L'ethnographie en migration(s)

\section{Femmes en mouvement, de nouvelles pistes pour repenser les migrations}

Compte rendu de la conférence de Camille Schmoll, cycle "l'Atelier du Savoir”, Espace Mendès France, Poitiers, 11 octobre 2018

Lydie Déaux, Alice Latouche et Benjamin Naintré

\section{CpenEdition}

Journals

Édition électronique

URL : https://journals.openedition.org/e-migrinter/1573

DOI : 10.4000/e-migrinter.1573

ISSN : 1961-9685

Éditeur

UMR 7301 - Migrinter

Référence électronique

Lydie Déaux, Alice Latouche et Benjamin Naintré, « Femmes en mouvement, de nouvelles pistes pour repenser les migrations », e-Migrinter [En ligne], 18 | 2019, mis en ligne le 01 mai 2019, consulté le 20 mai 2021. URL : http://journals.openedition.org/e-migrinter/1573 ; DOI : https://doi.org/10.4000/emigrinter.1573

Ce document a été généré automatiquement le 20 mai 2021.

Tous droits réservés 


\section{Femmes en mouvement, de nouvelles pistes pour repenser les migrations}

Compte rendu de la conférence de Camille Schmoll, cycle "l'Atelier du Savoir", Espace Mendès France, Poitiers, 11 octobre 2018

Lydie Déaux, Alice Latouche et Benjamin Naintré

\section{RÉFÉRENCE}

Schmoll, Camille (2018) Conférence « Femmes en mouvement, de nouvelles pistes pour repenser les migrations », Poitiers, Espace Mendès France.

1 Les ateliers du savoir, dispensés au centre Pierre Mendès France (Poitiers) en partenariat avec le laboratoire MIGRINTER, ont ouvert un cycle de conférences articulé autour de la question des femmes en exil. Intitulé «Femmes, migrations et engagements", sous la direction d'Adelina Miranda, professeure d'anthropologie (MIGRINTER, Université de Poitiers), et de Sarah Przybyl, post-doctorante et géographe (MIGRINTER), ces conférences entendent déconstruire la vision essentialisante des femmes comme victimes de la migration.

2 Au cours de cette première séance animée par Sarah Przybyl, intitulée «Femmes en mouvement. De nouvelles pistes pour repenser les migrations ", Camille Schmoll ${ }^{1}$ est revenue sur l'ensemble de ses recherches qui sont axées sur l'impact du genre dans les mouvements migratoires contemporains. L'objectif était de déconstruire certaines idées préconçues de la migration féminine, en passant par les figures idéal-typiques des femmes en exil, puis d'ouvrir de nouvelles pistes inscrites dans le champ de la géographie féministe. Toutefois, elle a tenu à souligner que l'outil du genre ne devait pas être exclusif, au risque d'occulter d'autres formes de domination.

3 La conception erronée qui revient régulièrement, lorsqu'on aborde la question de la migration des femmes, est qu'elles migreraient moins que les hommes. Or, les femmes 
ont toujours été présentes dans les flux migratoires, et sont aujourd'hui plus nombreuses que les hommes à vivre dans les pays « du Nord » (Catarino et Morokvasic, 2003). L'historienne Donna Gabaccia (2015) a démontré la présence ancienne des femmes, et ce dès les mouvements migratoires issus des colonisations. Cependant, jusqu'aux années 1980, la recherche en France et dans l'Europe accorde peu d'importance et d'intérêt aux migrations féminines. Pour comprendre cette invisibilité, il faut préciser que les analyses sociologiques sur les migrations ont longtemps privilégié l'homme comme un référent universel (Castles, De Haas, Miller, 2013). De même, les études féministes ont longtemps occulté la spécificité des femmes migrantes au profit de théories centrées sur le modèle d'une femme universelle exploitée par le patriarcat (Morokvasic, 2010). Ce cloisonnement des recherches a longtemps mis la situation des femmes migrantes dans une zone d'ombre. Toujours inclues dans une dynamique androcentrée, les femmes étaient considérées comme des "suivantes", rattachées à la figure d'épouse qui migrent par le biais du regroupement familial (Green, 2002). Cependant, cette voie n'est pas privilégiée par les femmes, qui migrent davantage de manière autonome, seules (Beauchemin, Borrel et Régnard, 2016).

Un second stéréotype récurrent, vis-à-vis de la migration féminine, est l'idée que le départ du pays d'origine aboutirait nécessairement à une émancipation et équivaudrait à une démarche moderne et libératrice (Miranda, 2010). Certes le processus migratoire transforme les rôles de genre et accélère les transformations de rapport de genre mais ne tend pas nécessairement vers une émancipation (Schmoll, 2005) : il convient donc davantage, comme le souligne Camille Schmoll dans ce séminaire, de parler d'«autonomie en tension", sans cesse collective et remodelée selon les relations sociales.

5 A l'inverse de ce modèle " héroïsé ", les femmes en exil sont souvent apparentées à des victimes dans l'imaginaire collectif. Cette représentation figée néglige les formes de résistances, de résilience qui existent même au sein d'un système d'oppression sexiste. Camille Schmoll a détaillé l'incarnation de ces résistances féminines qui prennent le corps comme support : certaines femmes n'hésitaient pas à recourir à la grève de la faim, ou refusaient de dormir sur des couchettes dans des centres de rétentions en Italie, pour marquer leur opposition aux conditions d'asile dans les centres d'accueil.

Enfin, les migrantes sont souvent qualifiées de mauvaises mères, épouses et filles, dès lors qu'elles quittent la cellule familiale et qu'elles s'arrachent au rôle traditionnel de femme assignée au foyer. Pour échapper à ces stigmates, les migrantes circulent entre leur pays d'origine et le pays d'arrivée pour conserver une attache avec leurs familles.

7 Il est néanmoins nécessaire de déconstruire ces stéréotypes en mettant en lumière les figures multiples des femmes migrantes, afin de souligner la complexité de leur expérience. Mais les recherches sur les exilées se confrontent à de nombreuses difficultés d'accès au terrain puisqu'il existe des logiques d'auto-invisibilisation: certaines femmes évitent les espaces publics et certains quartiers d'immigration pour ne pas être assimilées aux stigmates des populations migrantes.

8 Camille Schmoll se propose de décliner les figures « classiques » qui composent les flux migratoires des femmes en exil. La première d'entre-elles est celle de la travailleuse du care (Borgeaud-Garciandia et Georges, 2014 ; Falquet, Hirata et Lautier, 2006). Part conséquente des migrations internationales ${ }^{2}$, elle représente un aspect de l'économie mondialisée qu'on ne peut plus ignorer (d'où l'importance des travaux qui s'y consacrent). Dans le pourtour méditerranéen, il existe trois systèmes migratoires 
articulés autour de cette économie : d'abord le système proche-oriental qui repose sur la Kafala, un système de sponsorship par lequel les femmes migrent grâce à l'aide d'un sponsor, souvent une famille qui souhaite employer des migrantes pour réaliser un travail domestique. Il existe également un système sud-européen, composé de femmes d'Amérique latine, d'Espagne, d'Europe orientale et d'Asie qui travaillent dans l'aide aux personnes (personnes âgées et enfants) dans ce qu'on appelle «les chaines du soin ». Les pays d'Afrique du nord, enfin, sont marqués par une migration interne de femmes qui vont travailler dans des services domestiques, comme par exemple «les petites bonnes » au Maroc.

9 Les nombreuses recherches portées sur les métiers du care ne doivent pas cependant occulter les autres secteurs économiques des migrations féminines. La propension à émigrer augmente avec le niveau de qualification et c'est d'autant plus vrai pour les femmes spécialisées notamment dans le domaine de la santé ou des nouvelles technologies. Néanmoins, l'ensemble des travailleuses qualifiées connaissent un processus de déclassement dans les pays d'arrivée.

10 L'actualité migratoire de ces dernières années a rendu visible une deuxième figure des exilées, que Camille Schmoll nomme "les damnées de la mer " pour désigner ces femmes dont la route migratoire, à travers l'Afrique, la Lybie et la Méditerranée, est particulièrement longue, violente et mortelle. Potentiellement plus nombreuses que les hommes au départ, l'OIM les chiffre à 20-25\% du contingent migratoire à l'arrivée. Les politiques migratoires d'externalisation accroissent leur vulnérabilité sur la route les exposant davantage aux violences de genre tout au long de leur parcours, des passages aux frontières jusqu'à la traversée en mer durant laquelle elles sont plus nombreuses à mourir. Pourtant, ces violences ne sont pas prises en compte lors de l'évaluation des demandes d'asile qui ne regarde que celles subies au pays d'origine.

11 Camille Schmoll a terminé ce tour d'horizon des figures de la migration féminine par les travailleuses agricoles et les commerçantes. Leurs mobilités suivent des logiques de circulation pendulaire permettant un va-et-vient de biens matériels entre leur pays d'origine et les pays où elles font affaire dans le cas des commerçantes. En ce qui concerne les travailleuses agricoles, nous pouvons citer en exemple les programmes de recrutement circulaire au Maroc qui privilégient les femmes avec enfants pour éviter qu'elles fondent une famille une fois sur place.

12 Les migrations féminines sont donc plurielles. Mais pourquoi se restreindre aux femmes lorsque nous articulons genre et migration? Selon Camille Schmoll, il y a un intérêt fécond à faire dialoguer migrations féminines et masculines. Il continue d'être intéressant de s'intéresser à l'expérience spécifique des femmes en exil pour combattre les représentations androcentrées qui subsistent dans l'imaginaire collectif. Il s'agit de mettre en lumière par ces femmes d'autres échelles, d'autres lieux, d'autres dynamiques, afin d'inquiéter l'universel au masculin. Partir des parcours et croiser différentes dimensions (juridique, affective, géographique, sociale, etc.) permettent de donner à voir la complexité des ressorts, moteurs et contraintes des migrations féminines. Sur le plan méthodologique, une démarche longitudinale, c'est-à-dire les suivre physiquement et virtuellement, nous amène à penser leur parcours migratoire dans une dimension plus globale, celle de leur parcours de vie. Comment parlent-elles de leurs trajectoires? Comment ces femmes font famille en migration? Comment rendre compte des effets des rapports de pouvoir et de dominationsur leurs trajectoires? Privilégier les observations dans les espaces publics et s'inscrire dans une 
démarche de co-production des données rendraient opérante l'approche intersectionnelle dans son ambition de redonner voix aux femmes. La question du corps est ici centrale. Camille Schmoll nous l'a montré en diffusant un extrait vidéo de son terrain à Chypre auprès de travailleuses domestiques philippines. On les voit mettre en scène avec humour les arrestations policières qu'elles ont pu vivre et rendre ainsi visible l'expérience quotidienne des rapports de pouvoir dans l'espace public. Ces corps habituellement ethnicisés, racialisés, qui n'existent que dans la relation aux autres retrouvent ici une individualité témoignant d'une forme d'autonomisation permise par la transgression des rapports de genre. Ces derniers peuvent se lire, enfin, à travers les politiques migratoires qui reproduisent des schèmes de pensée genrés.

\section{BIBLIOGRAPHIE}

Beauchemin, Cris ; Borrel, Catherine ; Régnard, Corinne (2016) Chapitre 2. Hommes et femmes en migration : vers un rapprochement des profils et des trajectoires, In Beauchemin, C. ; Hamel, C. ; Simon, P. (dir.) Trajectoires et origines. Enquête sur la diversité des populations en France, Paris, Ined, 624 p. (Grandes Enquêtes).

Borgeaud-Garciandia, Natacha ; Georges, Isabel (dir.) (2014) Travail, femmes et migrations dans les Suds, Revue Tiers Monde, vol. 1, n²17, 248 p.

Castles, Stephen ; De Haas, Hein ; Miller, Mark J. (2013) The age of migration. International Population Movements in the Modern World, Basingstoke, Palgrave MacMillan, $401 \mathrm{p}$.

Catarino, Christine ; Morokvasic, Mirjana (2005) Femmes, genre, migration et mobilités, Revue Européenne des Migrations Internationales, vol. 21, n 1, pp. 7-27.

Falquet, Jules ; Hirata, Helena ; Lautier, Bruno (dir.) (2006) Travail et mondialisation. Confrontations Nord / Sud, Cahiers du Genre, n 40, 270 p.

Gabaccia, Donna R. (2015) Genre et migrations dans les études atlantiques de 1500 à nos jours, Revue européenne des migrations internationales, vol. 31, $\mathrm{n}^{\circ} 1$, pp. 15-37.

Green, Nancy (2002) Repenser les migrations, Paris, Presses universitaires de France, 138 p. (Le nœud gordien).

Miranda, Adelina (2010) Les multiples situations migratoires féminines dans la méditerranée, NAQD, vol. 1, n 28, pp. 21-34.

Morokvasic, Mirjana (2010) Des femmes au genre en migrations, NAQD, vol. 1, n 28, pp. 35-54.

Schmoll, Camille (2005) Pratiques spatiales transnationales et stratégies de mobilité des commerçantes tunisiennes, Revue Européenne des Migrations Internationales, vol. 21, $\mathrm{n}^{\circ} 1$, pp. 131-154.

Tayah, Marie-José (2016) Decent work for migrant domestic workers : moving the agenda forward, Rapport OIT, Genève, 108 p. 


\section{NOTES}

1. Maîtresse de conférences en Géographie à l'Université de Paris Diderot. Membre junior de l'Institut Universitaire de France et membre de l'UMR Géographie-Cités. HDR soutenu en 2017 à l'Université de Poitiers.

2. Selon l'OIT (Organisation Internationale du Travail), en 2013, les travailleuses migrantes du care étaient 8,5 millions et représentaient $73,4 \%$ du contingent migratoire dans ce secteur économique (OIT, 2015).

\section{INDEX}

Mots-clés : femmes, genre, exil, autonomie, expérience migratoire, relations entre communautés

\section{AUTEURS}

\section{LYDIE DÉAUX}

doctorante en sociologie, Laboratoire Migrinter (UMR 7301) CNRS / Université de Poitiers lydie.deaux@univ-poitiers.fr

\section{ALICE LATOUCHE}

doctorante en sociologie, Laboratoire Migrinter (UMR 7301) CNRS / Université de Poitiers et CRESPPA-GTM (UMR 7217), CNRS / Université Paris VIII Vincennes - Saint-Denis latouche.alice@gmail.com

\section{BENJAMIN NAINTRÉ}

doctorant en sociologie, Laboratoire Migrinter (UMR 7301) CNRS / Université de Poitiers benjamin.naintre@univ-poitiers.fr 